\title{
AN APPLICATION OF INTERFERENCE METHODS TO A STUDY OF THE CHANGES PRODUCED IN METALS BY MAGNETIZATION.
}

By J. S. Stevens.

DROBLEMS which have for their object a study of the changes produced in metals when magnetized have, during the past fifty years, received attention from physicists. It is thought that if a thorough investigation of the subject could be made, much light would be thrown upon the phenomenon of magnetization, and even the theory of the constitution of matter. Historical notes relating to this problem may be found in the Physical Review, Vol. III., No. I 5, and Vol. V., No. 5. The results of various investigators agree fairly well as to the maximum elongation of a rod or wire when saturated. There seems, however, to remain some difference of opinion concerning the behavior of rods under states of magnetization below the saturation point. If the general proposition that the change depends largely upon the kind and condition of the metal used may be assumed as correct, these discrepancies may be accounted for.

The important investigations made by Professor Rogers and others, in which interference methods were applied to the measurement of small temperature changes in the length of rods, suggested to me the possibility of applying similar methods to a study of this problem. The "interferometer" has now become so well known that it will not be necessary to describe it here. This instrument was used with slight modification of its construction. The mirror frame, which is commonly attached to the movable bed, was removed and firmly screwed to the end of the rod to be tested ( $A$ in the figure). The other end of the rod was made fast to a brass cylinder ( $K$ in the figure), which could be moved backwards and forwards, and thus provide the adjustment usually afforded when the mirror is moved by means of a micrometer screw. The magnetizing coil was 
supported at $S$ and the support as well as the interferometer were clamped in a slot so that adjustment in two directions was possible. The whole was attached to a heavy wooden frame, and little or no inconvenience was caused by vibrations in the building.

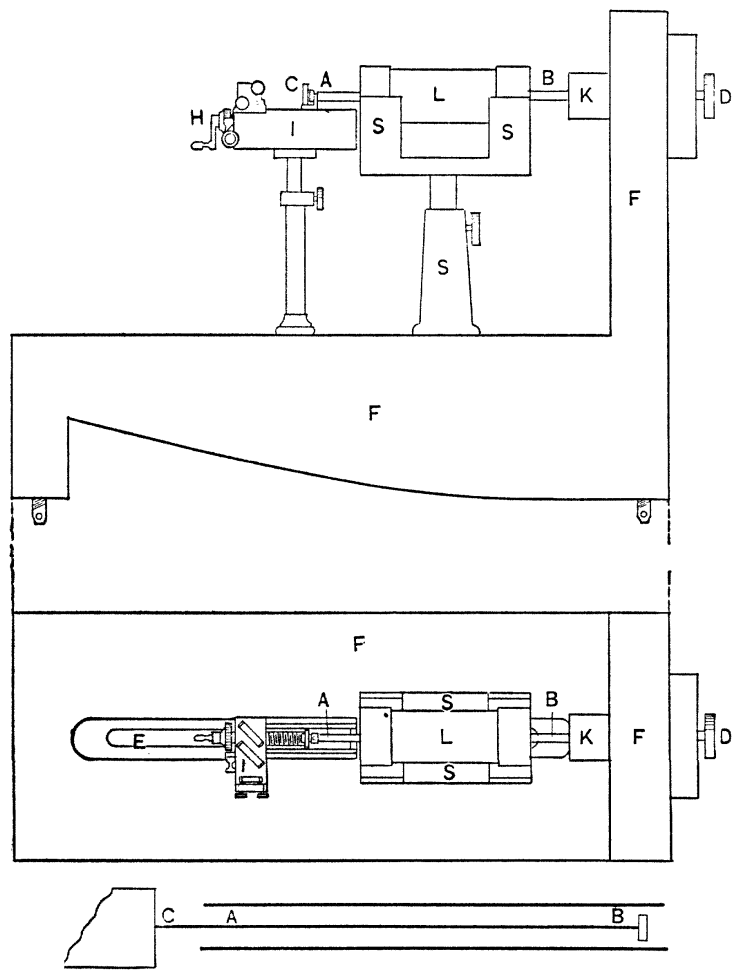

Fig. 1.

In the first part of the experiment, when it was desired principally to find the best working conditions and make some relative measurements, the precaution was not taken to make the coil longer than the rods, and the rods, while equal in diameter, were too large to afford the most satisfactory results. The coil was composed of I 5 layers of no. 25 copper wire and was $2 \mathrm{I} \mathrm{cm}$. long. It had a resistance of 420 ohms. Current was afforded by a storage battery of I IO volts. The rods were $30.5 \mathrm{cms}$. in length and $0.953 \mathrm{~cm}$. in diameter. Tests were made with wrought iron, cast iron, steel, copper, brass, nickel, and aluminum. 
Nature and Method of Micasurement.

The maximum elongation for soft iron wire observed by Dr. More (See Physical Review, Vol. III., No. I 5) was 0.000 $75 \mathrm{~cm}$. or about three wave-lengths of sodium light. By making the measurements therefore in wave-lengths we are provided with a direct method, and the measuring units are of about the same order of magnitude as the observed changes in length. When the mirror moves parallel to itself one-fourth of a wave-length, the cross wire of an observing telescope would appear to have passed over one uniformly colored space; if for example sodium light is used, one yellow space. The spaces may be easily read to tenths, or closer if one of the following methods is employed:

a. By using a micrometer microscope of proper focal distance and magnifying power.

b. By having a micrometer screw with a graduated head attached to the compensator.

$c$. By covering one half of the mirror with a scale which will divide the spaces into any desired number of parts.

By simple estimation the error of a single observation need not exceed I per cent., while a series of observations, employing one of the devices mentioned above would still farther reduce the error.

In case the change in the bar is sufficient to cause more than one fringe to pass the reference wire, fringes from white light should be employed. In this manner the identity of the fringes may be preserved.

$$
\text { Results. }
$$

The results obtained may be shown by the following tables which are followed by an explanation.

TABLE I.

SOFT IRON.

\begin{tabular}{|c|c|c|c|c|c|}
\hline II. & $I$. & $B$. & $R$. & $\begin{array}{c}\text { Maximum } \\
\text { Elongation. }\end{array}$ & $\partial l / l$ \\
\hline 5.5 & 183.0 & 2306 & 2920 & $0.020 \lambda$ & $4 \times 10^{-8}$ \\
\hline 17.6 & 689.6 & 8671 & 920 & $0.075 \lambda$ & 15 \\
\hline 31.1 & 977.8 & 12286 & 520 & $0.375 \lambda$ & 75 \\
\hline 34.4 & 1136.9 & 14296 & 470 & $0.425 \lambda$ & 85 \\
\hline 38.5 & 1343.3 & 16891 & 420 & $0.500 \lambda$ & 100 \\
\hline
\end{tabular}


TABLE II.

SOFT IRON.

\begin{tabular}{c|c|c}
\hline $\begin{array}{c}\text { No. of Successive } \\
\text { Readings. }\end{array}$ & Direction. & Amount. \\
\hline 1 & $e$ & $0.200 \lambda$ \\
1 & $e$ & $0.175 \lambda$ \\
1 & $e$ & $0.125 \lambda$ \\
1 & $e$ & $0.125 \lambda$ \\
10 & $e$ & $0.125 \lambda$ \\
2 & $c$ & $0.200 \lambda$ \\
6 & $e$ & $0.125 \lambda$ \\
2 & $c$ & $0.250 \lambda$ \\
5 & $e$ & $0.125 \lambda$ \\
10 & $c$ & $0.250 \lambda$ \\
4 & $e$ & $0.100 \lambda$ \\
1 & $e$ & $0.075 \lambda$ \\
1 & $c$ & $0.250 \lambda$ \\
3 & $e$ & $0.200 \lambda$ \\
5 & $e$ & $0.125 \lambda$ \\
1 & $c$ & $0.250 \lambda$ \\
\hline
\end{tabular}

TABLE III

JESSOP STEEL, RECTANGULAR SECTION.

\begin{tabular}{c|c|c|c}
\hline Resistance. & Elongation. & Resistance. & Elongation. \\
\hline 420 & $0.90 \lambda$ & 420 & $0.80 \lambda$ \\
470 & $0.60 \lambda$ & 470 & $0.40 \lambda$ \\
520 & $0.50 \lambda$ & 520 & $0.25 \lambda$ \\
620 & $0.45 \lambda$ & 620 & $0.15 \lambda$ \\
920 & $0.25 \lambda$ & 920 & $0.08 \lambda$ \\
1420 & $0.10 \lambda$ & & \\
1920 & $0.05 \lambda$ & & \\
& & & $0.15 \lambda$ \\
420 & $0.40 \lambda$ & 420 & $0.10 \lambda$ \\
470 & $0.25 \lambda$ & 470 & \\
520 & $0.15 \lambda$ & 520 & \\
620 & $0.05 \lambda$ & & \\
\hline 420 & $0.10 \lambda$ & & \\
\hline 470 & $0.05 \lambda$ & & \\
\hline
\end{tabular}


In Table I. the attempt was made to find what relation existed between the magnetizing force and the magnetic intensity, and the elongation. As these values were in no case maximum values, the table merely shows that the latter increases with the former for the quantities used.

In making the observations for Table I. it was noticed after several successive readings had been taken that the behavior of the rod was somewhat abnormal. For example, after several elongations there would follow a contraction, and there seemed to be no method of predicting the nature or extent of the change. These occurred only after quite a number of readings which were regular. As these results were quite unexpected I tried the experiment on several occasions, and had the observations checked by an assistant. While no uniformity existed among the different readings, the abnormal action was always noted. In Table II., I have collected the results of a series of observations. Column I represents the number of observations which were uniform both in magnitude and direction; column 2 states the nature of the change-whether an elongation or a contraction; column 3 shows the magnitude of the change.

Table III. represents the results when currents decreasing in magnitude were successively sent around a rectangular bar of Jessop steel. Resistances varying from 420 to 1920 were placed in the circuit and the elongations noted ; then these measurements were immediately repeated, so that in all five series were observed. The results obtained show :

a. Considerable regularity in the relation between current and elongation.

b. A regular decrease in elongation for the same current after repeated applications.

c. No abnormal effects as in the case of soft iron.

\section{Distortion.}

By use of interference methods, changes which might occur in a rod other than expansion or contraction may be noted. For example, if the rod is distorted the effect is very clearly seen. If the distortion is the result of horizontal planes slipping past each other 
in a direction parallel to the length of the rod, the mirror will move as though rotated around a horizontal axis. In this case the fringes, which formerly appeared vertical, become inclined at an angle depending upon the amount of distortion. If the distortion is caused by a slipping of vertical planes, the mirror appears to rotate on a vertical axis, and the fringes approach or recede from each other. The first effect was quite noticeable in the case of soft iron, but did not appear in steel or nickel. The second was suspected in several observations but was more difficult to detect. No error is introduced in the method on account of the distortions as the extreme displacement of the fringes due to this cause would correspond with the maximum elongation or contraction sought.

Aluminum, copper, and brass gave no results that could be detected. Nickel showed a contraction for the values of $H$ used of about $i / 4$. Upon making and breaking the circuit repeatedly it exhibited the abnormal action noticed in the case of soft iron. Cast iron expanded slightly and quite uniformly.

A test was made to determine whether the effect of change in temperature introduced an error. Bars of non-magnetic substances mentioned above showed no change in length due to heating effects, although a charge of $\lambda / 40$ could readily have detected. Again the circuit was allowed to remain closed for some time until a change in temperature sufficient to be noticed resulted. The change was a creping motion of the fringes which was in strong contrast with the sudden change observed when the bar was magnetized.

The initial contraction in metals which expand on magnetization, which has been reported by some observers, was not detected in this experiment. If it exists at all it must be characteristic of rods under certain conditions of stress, or containing certain peculiarities of structure not exhibited by the rods here used.

Having in the above investigations determined the best working conditions, and studied the behavior of various rods and the peculiarities of their state when magnetized, the experiment was assigned to a senior in the University, Miss Rena E. Dunn, to repeat the measurements under more favorable conditions. Dimensions were employed as follows: 

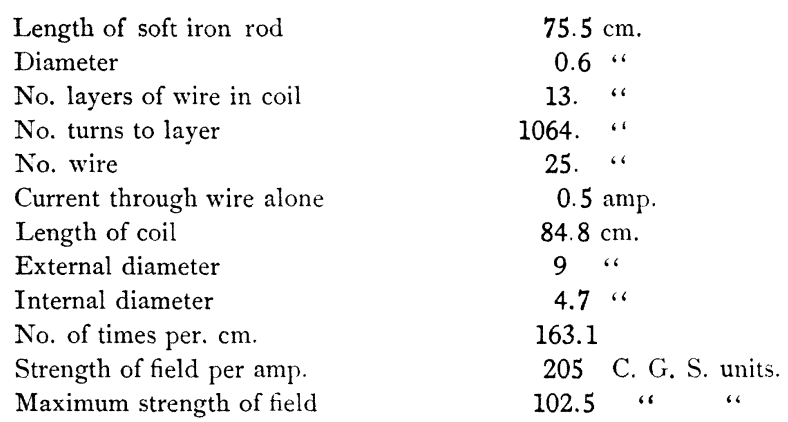

The coil being $9.3 \mathrm{~cm}$. longer than the rod it may be assumed that the magnetizing force was used to good advantage in magnetizing the rod. As shown in the drawing the mirror was fastened on one end of the rod and placed within the coil, while the other end of the rod was joined to a strip of brass $A C$ in the smaller figure, which in turn connected with the movable cylinder. For convenience a special frame was made for the remaining mirrors of the interferometer, but a description of this would not be necessary here. The current, as before, was attained from a I IO-volt storage battery and was measured simultaneously with the optical measurements, by means of a Weston milli-voltmeter with a specially constructed shunt. Variation in resistance was produced by a bank of lamps.

In the following Table (IV.) al represents the mean of twenty observations in the first case, and ten in each succeeding case with probable errors averaging $0.1 \%$.

TABLE IV.

\begin{tabular}{l|r|r|r|r|c}
\hline $\begin{array}{l}\text { Current } \\
\text { (Amps.) }\end{array}$ & \multicolumn{1}{|c|}{$H}$. & \multicolumn{1}{|c|}{$I}$. & \multicolumn{1}{|c|}{$B}$. & $\partial l$ & $\partial l / l$ \\
\hline 0.500 & 102.5 & 1835.4 & 23173.1 & $13.37 \times 10^{-5} \mathrm{~cm}$. & $17.7 \times 10^{-7}$ \\
0.185 & 41.0 & 1153.2 & 14537.2 & 6.79 & 8.9 \\
0.096 & 20.5 & 813.7 & 10249.1 & 4.66 & 6.2 \\
0.061 & 12.8 & 620.6 & 7813.9 & 2.42 & 3.2 \\
0.043 & 9.3 & 510.8 & 6430.3 & 1.55 & 2.1 \\
0.028 & 5.7 & 446.9 & 5626.1 & 1.18 & 1.6 \\
0.022 & 4.7 & 420.5 & 5290.3 & 1.11 & 1.5 \\
\hline
\end{tabular}

Comparing these values for $a l l$ with those obtained by Dr. More, it will be noticed that for small values of $H$ the relative elongations 
were much greater; while as $H$ increased the elongations failed to increase in an equal ratio. His maximum was reached when $H=37.6$, while in this case for $H=102.5$ the elongation surpassed that for any smaller value.

In conclusion the following may be claimed as points of advantage passed by this method :

I. Since the mirror is practically a part of the rod, little or no annoyance due to vibrations of the building was experienced.

2. The measurements are direct and the unit so small that the error need not exceed one per cent.

3. The errors liable to occur in any mechanical device, such as the use of levers, indicators and the like, are eliminated.

4. Any auxiliary effects, such as distortion, are closely seen.

In addition to the work of Miss Dunn already mentioned, I ought to state that most of the mechanical arrangements for this investigation were made by Mr. L. H. Horner of the class of 1900 , who also assisted me greatly in taking the observations.

Physical Laboratory, University of Maine. 